\title{
Investigations on a MOX Gas Sensor as an Infrared Source for an IR-based Gas Sensing System
}

\author{
K. Kühn, M. Leidinger, E. Pignanelli, A. Schütze \\ Saarland University, Department of Mechatronics, Laboratory for Measurement Technology, \\ Campus A5 1, 66123 Saarbruecken, Germany, \\ k.kuehn@LMT.uni-saarland.de
}

\begin{abstract}
:
In this work a micro structured metal oxide (MOX) gas sensor was investigated as an infrared (IR) radiation source for an IR gas measurement system. Thus, the advantages of both sensor principles can be combined, namely the high sensitivity and broad response spectrum of the MOX gas sensor based on a chemical sensing principle as well as the high selectivity and stability of the IR measurement based on a physical sensing principle. This selectivity for one application specific gas molecule is achieved by a radiation sensor with integrated optical filter. For example, for indoor air quality monitoring the IR measurement could be used to measure the concentration of carbon dioxide $\left(\mathrm{CO}_{2}\right)$, while the MOX gas sensor could be used for detecting volatile organic compounds (VOCs).
\end{abstract}

Key words: Metal oxide (MOX) gas sensor, IR gas measurement, chemical sensing principle, physical sensing principle, indoor air quality monitoring.

\section{Introduction}

Modular gas sensing systems based on application specific combinations of different sensor principles are gaining great interest. Limitations with respect to detection limits for different gases result mostly out of restrictions caused by cost-benefits reflections. The investigations in this work aim at replacing a comparatively cost-intensive IR-source for an existing NDIR/PAS combination with inherent self monitoring [1] with a MOX gas sensor. Thus, the number of components remains constant and the total cost decreases while the information about the ambient gas composition increases notably, especially making use of temperature cycled operation (TCO) for evaluation of the MOX gas sensor signal [2].

\section{Fundamentals}

In addition to low power consumption and a short time constant a micro structured MOX gas sensor offers considerable advantages of low cost, high sensitivity (down to ppb detection limit) and long life. Due to the temperature cycled operation the known disadvantages of poor selectivity and drift of the sensor signal can be resolved. The measured sensor response is based on the gas dependent DC resistance of the metal oxide gas sensing layer, which is additionally modulated by the changing temperature resulting in gas specific response patterns. In addition, TCO modulates the IR intensity emitted from the sensor, which is required for the IR gas measurement system. In contrast to the chemical sensing principle of the MOX, the physical sensing principle is based on the gas specific absorption of IR radiation. We make use of a combined transmission/ absorption IR measurement, in which the transmission is registered with a thermopile radiation sensor. The absorbed IR energy leads to an excited state with increased vibrational energy of the gas molecules that is mainly converted into heat, increasing the local temperature and thus the overall pressure, due to the constant gas volume [3]. This photoacoustic effect (PAE) shows inverse behavior compared to the transmission signal, i.e. increasing signal with increasing gas concentration. The main advantages of the IR measurement are high stability and accuracy, the combination of transmission and PAE allows an intrinsic self-monitoring of the system without requiring a second IR reference channel. In this work, only results of the IR transmission are presented as the gas specific filter was placed after the gas chamber preventing efficient PAS excitation. 


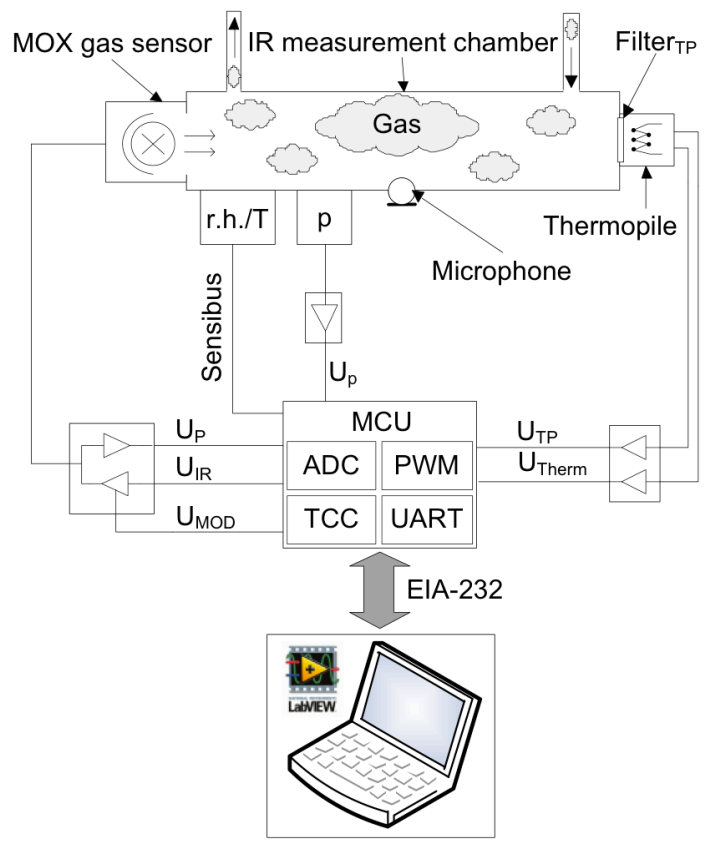

Fig. 1: Schematic for the measurement setup to verify the use of a conventional MOX gas sensor (MICS5131, e2v) as IR source for a thermopile sensor with $\mathrm{CO}_{2}$-filter (HIS A21 F4.26, Heimann Sensor).

\section{Experimental setup}

Using the measurement setup shown in Fig. 1 a MOX gas sensor (MICS5131, e2v) has been evaluated as IR source combined with a thermopile sensor with $\mathrm{CO}_{2}$-selective filter (HIS A21 F4.26, Heimann Sensor; center wavelength $4.26 \mu \mathrm{m})$. The whole measurement system, including also sensors for monitoring of ambient conditions (relative humidity r.h., temperature $T$, pressure $p$ ) as well as the required electronics is controlled by a microcontroller. Configuration settings and data acquisition are set in a graphical user interface (GUI), programmed with LabVIEW. The communication between the host PC (LabVIEW interface) and the microcontroller is based on the EIA-232 standard. The signal evaluation of the thermopile's raw data is performed offline using Matlab and is based on the calculation of discrete Fourier transformations (DFT) to achieve a good signal to noise ratio (SNR). For quantitative analysis the squared magnitude of the Fourier component at the excitation frequency $\left(1^{\text {st }}\right.$ Fourier components $)$ over 90 square wave temperature cycles of the MOX gas sensor heater is calculated and used to determine the calibration curve vs. gas concentration. Concerning the MOX sensor's raw data no further signal evaluation is performed in this study, but the usual proven signal processing steps can be applied for gas

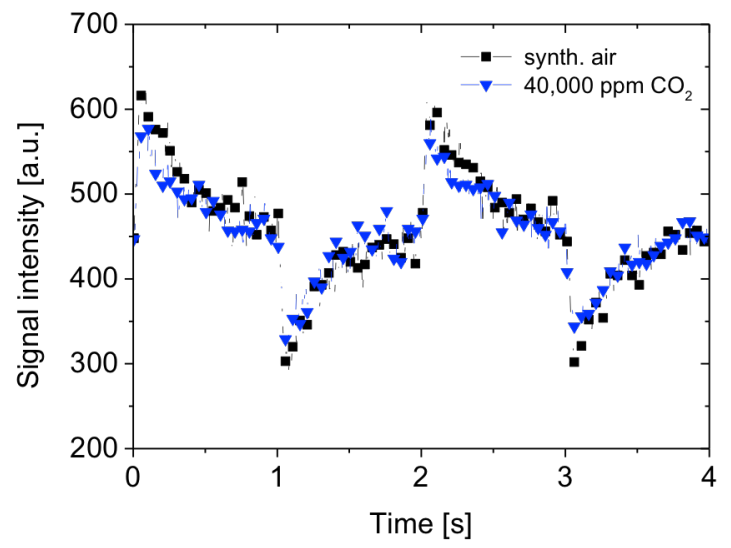

Fig. 2: Extracted raw data from thermopile sensor (0.5 Hz square wave modulation of the MOX gas sensor, 10 bit resolution, sampling rate $200 \mathrm{~Hz}$ ) for synthetic air and 40,000 ppm $\mathrm{CO}_{2}$ concentration.

identification and determination of the concentration [2].

\section{Results}

In the following, first results of both signal evaluations (thermopile and MOX gas sensor signal) are presented. Fig. 2 shows the thermopile raw data for synthetic air as well as for a $\mathrm{CO}_{2}$ concentration of $40,000 \mathrm{ppm}$, when operating the integrated heater of the MOX gas sensor with the maximum heater power of approx. $100 \mathrm{~mW}$ at $0.5 \mathrm{~Hz}$ (square wave modulation). The shape of the signal intensity of the thermopile raw data (10 bit $A D C$ ) results from an implemented electronic bandpass circuitry and its amplification must be adapted, depending on the application. Even if there is no obvious difference in the curves in Fig. 2, the thermopile's signal evaluation with DFT analysis as described above allows clear discrimination of the signals to determine the

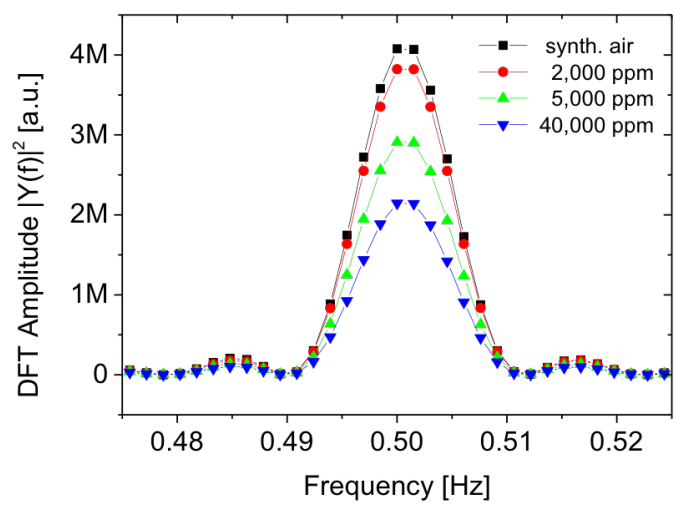

Fig. 3: DFT spectra for different $\mathrm{CO}_{2}$ concentrations showing the correlation between gas concentration and $1^{\text {st }}$ Fourier component. 


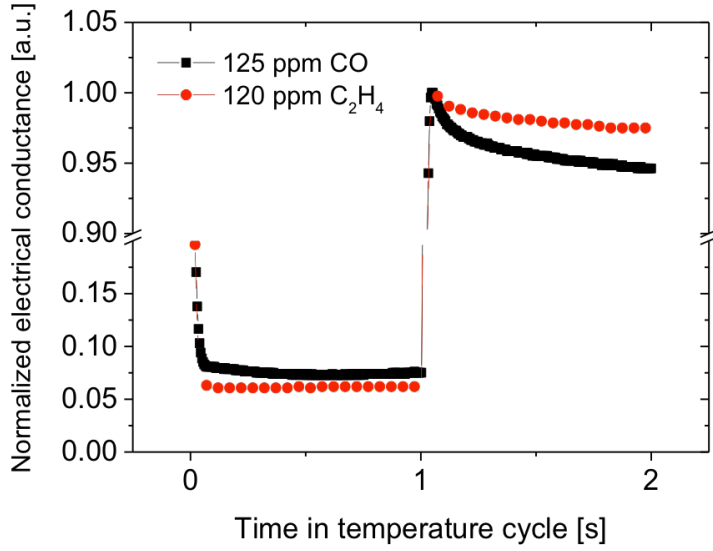

Fig. 4: MOX gas sensor response showing the normalized conductance of the gas sensing layer for $125 \mathrm{ppm} \mathrm{CO}$ and $120 \mathrm{ppm} \mathrm{C}_{2} \mathrm{H}_{4}$ obtained within one square wave modulated temperature cycle, normalized to the cycle's maximum value. The shape of the sensor response can be used for gas identification, while the maximum value can be evaluated for quantification of the gas concentration.

gas concentration. In the first setup described here, the detection limit is approx. 1,000 ppm (cf. Fig. 3) and a higher resolution can be achieved with further optimization of the system or longer measurement as described in [4]. Fig. 3 shows the squared DFT amplitude vs. frequency for three different $\mathrm{CO}_{2}$ concentrations compared to pure synthetic air. Thus, determination of the $\mathrm{CO}_{2}$ concentration requires $3 \mathrm{~min}$ (90 TCO cycles of $2 \mathrm{sec}$. each), which is adequate for indoor air quality applications. Fig. 4 shows exemplary response patterns of the MOX gas sensor. The normalized electrical conductance of the gas sensing layer is shown

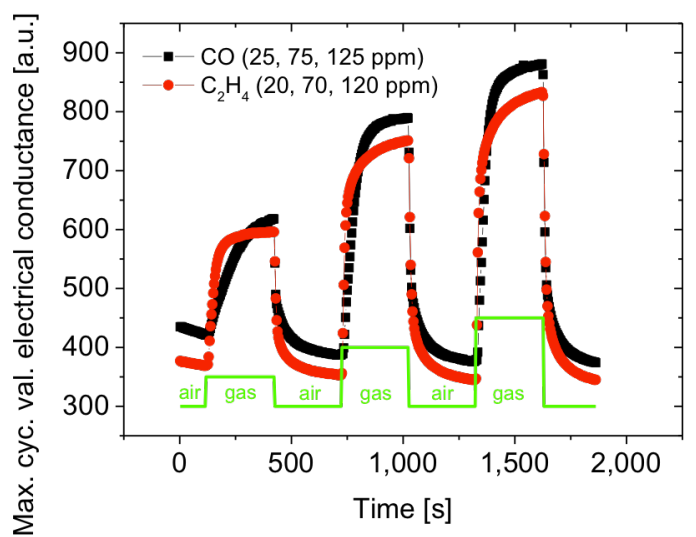

Fig. 5: Maximum value of the conductance over one cycle $(0.5 \mathrm{~Hz}$ square wave modulation) of the temperature cycled MOX gas sensor signal for two gases $\left(\mathrm{CO}, \mathrm{C}_{2} \mathrm{H}_{4}\right)$. The sensor was exposed to three concentrations of each gas for $300 \mathrm{~s}$ and the measurement chamber was purged with synthetic air between the gas exposures, also for $300 \mathrm{~s}$. for two different gases for one temperature cycle $(0.5 \mathrm{~Hz}$ square wave modulation). The shape of the sensor response can be used for gas identification [2], while the signal magnitude, i.e. the normalization factor or the maximum value over the temperature cycle could be evaluated for quantification of the gas concentration. Fig. 5 presents the temperature cycle's maximum value over time for different gas concentration of $\mathrm{CO}_{2}$ and ethene $\left(\mathrm{C}_{2} \mathrm{H}_{4}\right)$. Both gas concentrations show different behavior to higher concentrations. For $\mathrm{CO}_{2}$ the starting time for steady state conditions of the curve is reciprocally proportional to higher concentrations, whereas for $\mathrm{C}_{2} \mathrm{H}_{4}$ the time increases with higher concentration. Especially to overcome the problem concerning the required long measurement time in Fig. 5, drift of the sensor response etc., the MOX gas sensor signal evaluation as described in [2] is a promising approach.

In the frame of this novel approach with a MOX gas sensor in the temperature cycled operation (TCO) as an IR radiation source, a correlation between the carrier gas and the thermopile signal could be observed. In the following the DFT evaluated signal of the thermopile sensor with $\mathrm{CO}_{2}$ filter at the excitation frequency $\left(1^{\text {st }}\right.$ Fourier component) over 90 consecutive TCO cycles of the MOX sensor $(100 \mathrm{~mW}, 6 \mathrm{~Hz}$ square wave modulation) was investigated for different gas compositions and concentrations (e.g. $\mathrm{C}_{2} \mathrm{H}_{4}, \mathrm{NH}_{3}, \mathrm{NO}, \mathrm{NO}_{2}, \mathrm{CO}$ ) at $75 \%$ relative humidity. The modulation frequency of the MOX gas sensor was changed to $6 \mathrm{~Hz}$ in order to select a trade-off between the thermopile's

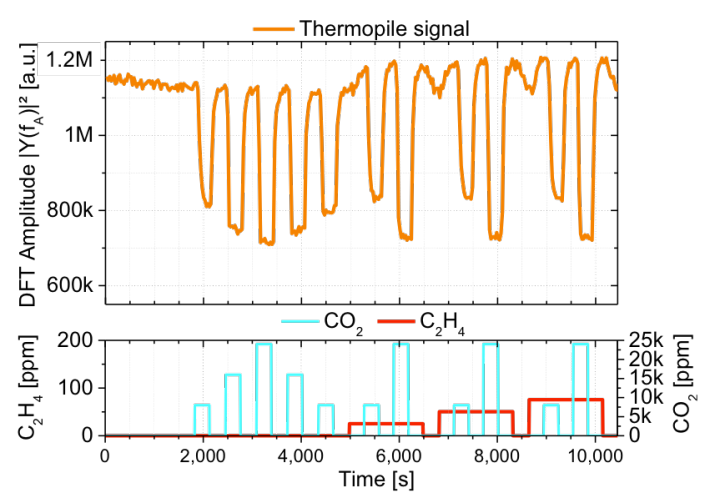

Fig. 6: Top, DFT evaluated thermopile signal at the excitation frequency $\left(f_{A}=6 \mathrm{~Hz}\right.$, square wave modulation, sampling rate $330 \mathrm{~Hz}, 100 \mathrm{~mW}$ ) for different $\mathrm{CO}_{2}$ concentrations based on the MOX gas sensor as IR source. A shift of the baseline is observed when $\mathrm{C}_{2} \mathrm{H}_{4}$ is additionally introduced. The measurement chamber was purged with synthetic air between the gas exposures. The applied gas profile for cross sensitivity investigations between gas concentration and detected radiation is shown at the bottom. 
modulation depth and the microphone's frequency characteristic. Thus, the resulting measurement period takes only $15 \mathrm{~s}$. Fig. 6 shows the evaluated thermopile signal in the upper graph, while the applied gas concentrations are shown in the lower graph. First a $\mathrm{CO}_{2}$ step profile $(8 \mathrm{k}, 16 \mathrm{k}, 24 \mathrm{k}, 16 \mathrm{k}, 8 \mathrm{k}$ ppm) was applied. The concentration can easily be determined by the thermopile sensor. When $\mathrm{C}_{2} \mathrm{H}_{4}$ is added at concentrations of $25,50,75$ ppm, respectively, the evaluated thermopile signal shows a changing of its baseline which corresponds to the electrical conductance of the MOX gas sensor (cf. Fig. 5) and, at the same time, the amplitude of the thermopile sensor response shows a slight increase from 400 to 450 a.u. for $24,000 \mathrm{ppm} \mathrm{CO}_{2}$ compared to the measurement without ethene. This behavior could be the result of a temperature change of the MOX gas sensor. However, we think that this signal change is caused by a gas induced change of the IR emissivity of the MOX sensing layer. In contrast to the effect caused by exposure to $\mathrm{C}_{2} \mathrm{H}_{4}$ as a reducing gas, i.e. the electrical conductance of gas sensing layer increases, the opposite behavior was observed for an oxidizing gas like nitrogen monoxide (NO). Fig. 7 shows this inverse behavior. When applying $\mathrm{NO}(50,100,150 \mathrm{ppm})$ the baseline of the evaluated thermopile signal is reduced remarkably and the relative amplitude of the sensor response for $\mathrm{CO}_{2}$ detection is also reduced (approx. 350 a.u. for the highest $\mathrm{CO}_{2}$ concentration applied). Note that the IR baseline remains low even when the chamber is purged with synthetic air. This correlates to the MOX sensor conductance, which only slowly returns to its normal value after exposure to NO. For both $\mathrm{C}_{2} \mathrm{H}_{4}$ and $\mathrm{NO}$, the baseline shift observed for the IR signals was almost

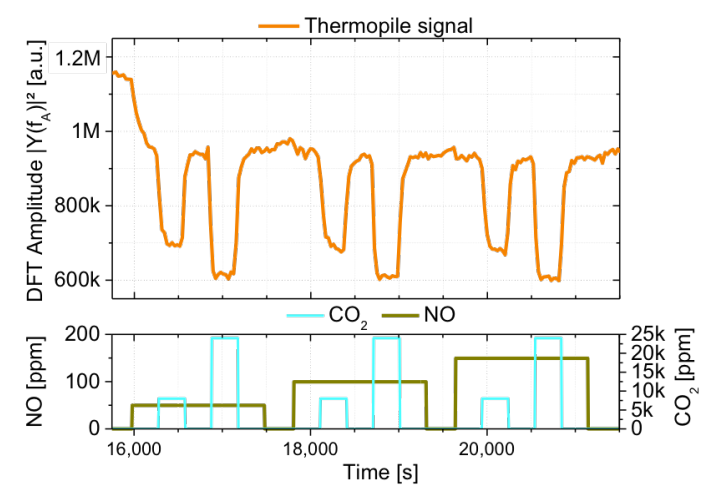

Fig. 7: Top, DFT evaluated thermopile signal at the excitation frequency of the temperature cycled MOX gas sensor for different $\mathrm{CO}_{2}$ concentrations with and without addition of NO, in analogy to Fig. 6. The applied gas profile is again shown at the bottom. independent of the gas concentration, which would indicate that the effect on the sensor conductance and the suspected influence on the emissivity are not proportional.

\section{Conclusion and Outlook}

To achieve comparable sensitivity for the absorption (PAE) signal as for the transmission (thermopile) signal a new design of the measurement setup has been developed placing the gas specific filter between two measurement chambers. The first chamber contains the MOX gas sensor, the second is used for the combination of IR-transmission/absorption measurements. Furthermore, a tunable, micro structured Fabry-Pérot filter [5] between both measurement chambers will provide a larger range of target gases for the IR sensor system [3]. Concerning the observed influence of trace gases to the IR signal further experiments are required to determine the cause, e.g. changes of the MOX gas sensors emissivity due to gas adsorption. For the signal evaluation this cross sensitivity must be adjusted. However, this unwanted effect could provide a basis for self-monitoring of the sensor system. Finally, the influence of ambient conditions, e.g. temperature, relative humidity and pressure, needs to be elaborated to achieve reliable quantitative results.

\section{Acknowledgements}

The authors want to thank two students ( $P$. Bost and C. Gerlach) that have contributed to this work with measurements studying the cross sensitivity concerning different trace gases within the scope of a small lab project

\section{References}

[1] K. Kühn, E. Pignanelli, A. Schütze, Kombinierte NDIR-PAS-Gasmessung mit großem Dynamikbereich und inhärenter Selbstüberwachung, 10. Dresdner Sensor Symposium, 2011, ISBN 978-3-942710-53-4, S. $51-54$.

[2] A. Schütze, A. Gramm, T. Rühl, Identification of Organic Solvent by a Virtual Multisensor System With Hierarchical Classification, IEEE Sens. J., Vol. 4, No. 6, 857-863 (2004), doi: 10.1109/JSEN.2004.833514.

[3] K. Kühn, M. Siegwart, E. Pignanelli, T. Sauerwald, A. Schütze, Versatile Infrared Gas Measurement System with tunable Microstructured Fabry-Pérot Filter, accepted IEEE I²MTC 2012.

[4] E. Pignanelli, K. Kühn, A. Schütze, Versatile Gas Measurement System Based on Combined NDIR Transmission and Photoacoustic Spectroscopy, IEEE Sensors 2011, doi: 10.1109/ICSENS. 2011.6127048.

[5] N. Neumann, M. Ebermann, K. Hiller, S. Kurth, Tunable infrared detector with integrated micromachined Fabry-Perot filter, J. Micro/Nanolith. MEMS MOEMS 7, 021004 (Apr 30, 2008), doi: 10.1117/1.2909206. 